\title{
FINITELY PRESENTED ORDERED GROUPS
}

\author{
by A. M. W. GLASS*
}

(Received 7th February 1989)

Theorem. There exist non-Abelian finitely presented lattice-ordered groups which are totally ordered. This disproves a previous conjecture of the author [5].

1980 Mathematics subject classification (1985 Revision): 06F15.

A group $G$ with a partial order on it that satisfies $(a \leqq b \rightarrow f a g \leqq f b g)$ for all $a, b, f, g \in G$ is called a p.o. group. If the partial order is a lattice (for every $a, b \in G$, there is a least upper bound $a \vee b$ and a greatest lower bound $a \wedge b$ ) the p.o. group is said to be a lattice-ordered group, or l-group for short. A p.o. group in which the partial order is total (for all $a, b, a \leqq b$ or $b \leqq a$ ) is called an o-group.

The class of $l$-groups is an equationally defined class of algebras under the operations $\cdot,^{-1}, \vee$ and $\wedge$. Hence free $l$-groups on arbitrary sets $X$ exist [2, Chapter IV].

If $G$ is an $l$-group and $H$ is a subgroup of $G$ that is closed under the lattice operations $\checkmark$ and $\wedge$, we call $H$ an $l$-subgroup of $G$. A homomorphism (embedding, isomorphism) between $l$-groups that preserves the lattice and group operations is said to be an $l$ homomorphism (l-embedding, l-isomorphism). The kernels of $l$-homomorphisms are precisely the convex normal $l$-subgroups ( $C$ is said to be convex in $G$ if $x \in G, c_{1}, c_{2} \in C$ and $c_{1} \leqq x \leqq c_{2}$ imply $x \in C$ ). If $K$ is a convex normal $l$-subgroup of $G$, then $G / K$ is an $l$-group under the naturally induced order $(K f \leqq K g$ iff $h f \leqq g$ for some $h \in K)$; see [1,Section 2.3] where, as usual, iff is shorthand for if and only if. If an l-group $G$ contains an Abelian convex normal $l$-subgroup $A$ such that $G / A$ is Abelian, then $G$ is said to be l-metabelian.

An l-group $G$ is said to be finitely presented (as an l-group) if there is a finite set $x_{1}, \ldots, x_{m}$ and a finite set $w_{1}, \ldots, w_{n}$ of elements of the free $l$-group $F$ on $\left\{x_{1}, \ldots, x_{m}\right\}$ such that $G$ is $l$-isomorphic to $F / N$ where $N$ is the convex normal $l$-subgroup of $F$ generated by $w_{1}, \ldots, w_{n}$. In this case we simply write $\left\langle x_{1}, \ldots, x_{m} ; w_{1}=e, \ldots, w_{n}=e\right\rangle$ for $F / N$, where throughout $e$ denotes the identity element of a group. The set $\left\{w_{1}=\right.$ $\left.e, \ldots, w_{n}=e\right\}$ is called the set of (defining) relations for $F / N$.

In any $l$-group $G$, let $|g|=g \vee g^{-1}$ for $g \in G$. It is easy to see [1, 1.3.10 and 1.3.11] that $|g| \geqq e$, and $|g|=e$ iff $g=e$. Hence $\left(w_{1}=e \& \ldots \& w_{n}=e\right)$ iff $\left|w_{1}\right| \vee \cdots \vee\left|w_{n}\right|=e$; thus any

\footnotetext{
* Research supported in part by an NFS U.S.-U.K. grant. I am extremely grateful to the NSF for making this research possible and to members of the Department of Pure Mathematics and Mathematical Statistics, and especially to John Wilson, for their hospitality and assistance.
} 
finitely presented $l$-group can be given by a single defining relation and so is an $m$ generator one relator $l$-group for some finite $m$.

In [5] we conjectured that the only finitely presented $l$-groups that are $o$-groups are $\mathbb{Z}$, the additive group of integers under the usual ordering, and $\{e\}$; also, that the only finitely presented $l$-groups that are subdirect products of $o$-groups are Abelian. This was shown to be the case if the defining $w_{1}, \ldots, w_{n}$ were all group words, see [3]. However, in this note we prove both conjectures are false with an easy example.

Theorem. There is a countably infinite set of pairwise non-l-isomorphic two generator one relator l-metabelian non-Abelian o-groups.

Clearly there are only countably many finitely presented l-groups. Moreover, one generator l-groups are Abelian and free l-groups on at least two generators are not subdirect products of $o$-groups. Hence the theorem is the best (or worst?) possible.

Throughout we use $\mathbb{Q}$ for the additive group of rationals with the usual order; $A \overline{\times} B$ for a semidirect product of $A$ by an $o$-group $B$ where $a_{1} b_{1} \leqq a_{2} b_{2}$ iff $b_{1}<b_{2}$ in $B$ or both $b_{1}=b_{2}$ and $a_{1} \leqq a_{2}$ in $A$; and $a \ll b$ for $a^{n} \leqq b$ for all $n \in \mathbb{Z}$.

For any further background, see $[1,4,5]$ if necessary.

We first give a permutation proof in outline and then provide a more formal proof in detail.

Permutation Proof. Let $m>1$ be a positive integer and $g$ be the order-preserving permutation of the real line given by: $\alpha \mapsto \alpha+1$. Then there are order-preserving permutations $f$ of the real line conjugating $g_{0}$ to $g_{0}^{m}$ but for any such $f$, there are real numbers $\alpha$ and $\beta$ such that $\alpha f<\alpha$ and $\beta f>\beta$ (see [4, Lemma 2.2.1]). Hence if $f$ and $g$ are any order-preserving permutations of the real line that move no point down and $f^{-1} g f=g^{m}$, then $g$ has infinitely many intervals of support and $f$ moves each interval of support of $g$ to one strictly to the right. Consequently, $g \ll f$. If $L(m)$ is the $l$-subgroup generated by $f$ and $g$, then the normal subgroup $C_{m}$ of $L(m)$ generated by $g$ is convex and Abelian. Moreover, it is an $o$-group whence $L(m)$ is an $l$-metabelian $o$-group. Since every countable $l$-group can be $l$-embedded in the $l$-group of all order-preserving permutations of the real line [4, Corollary $2 L], L(m) \cong\left\langle x, y ; x^{-1} y x=y^{m}, x \wedge y=y\right.$, $y \wedge e=e\rangle$. Clearly $L\left(m_{1}\right) \cong L\left(m_{2}\right)$ iff $m_{1}=m_{2}$. The theorem follows.

Proof of Theorem. Let $m$ be a positive integer exceeding 1 and

$$
L_{m}=\left\langle x, y ; x^{-1} y x=y^{m}, x \wedge y=y, y \wedge e=e\right\rangle \text {. }
$$

So $L_{m}$ is a finitely presented $l$-group for each $m$. We will prove that $L_{m}$ is actually an l-metabelian o-group.

By definition, $y \leqq x$. If $y^{n} \leqq x$ then $y^{m+n} \leqq x y^{m}=y x$; hence $y^{n+1} \leqq y^{m+n-1} \leqq x$ since $m \geqq 2$ and $y \geqq e$. Thus $y^{n} \leqq x$ for all integers $n$ by induction; so $y \ll x$. Consequently, $x^{-j} y x^{j} \ll x$ for all integers $j$. So if $C_{m}$ is the normal $l$-subgroup of $L_{m}$ generated by $y$, then $C_{m}$ is convex; clearly it is Abelian. 
We now examine $C_{m}$. We first note that

$$
x^{j} y^{i} x^{-j} \leqq x^{s} y^{r} x^{-s} \text { iff } i / m^{j} \leqq r / m^{s} .
$$

For if $j \leqq s$, then $x^{j} y^{i} x^{-j} \leqq x^{s} y^{r} x^{-s}$ iff $x^{-(s-j)} y^{i} x^{s-j} \leqq y^{r}$ iff $y^{i m^{s-j}} \leqq y^{r}$ iff $y^{i m^{s}} \leqq y^{r m^{j}}$ iff $i m^{s} \leqq r m^{j}$; similarly if $s \leqq j$. Moreover,

$$
x^{j} y^{i} x^{-j} \cdot x^{s} y^{r} x^{-s}=\left\{\begin{array}{lll}
x^{s} y^{i m^{s-j+r}} x^{-s} & \text { if } & j \leqq s \\
x^{j} y^{i+r m^{j-s}} x^{-j} & \text { if } & s \leqq j
\end{array}\right.
$$

Therefore if $\phi: C_{m} \rightarrow \mathbb{Q}$ is given by: $\left(x^{j} y^{i} x^{-j}\right) \phi=i / m^{j}$, then $\phi$ is an embedding and $z \leqq t$ iff $z \phi \leqq t \phi$ for all $z, t \in C_{m}$. Consequently $C_{m}$ is an Abelian o-group.

Each element of $L_{m}$ has the form $w x^{k}$ for some $w \in C_{m}$ and unique integer $k$. Furthermore $w_{1} x^{j} \leqq w_{2} x^{k}$ iff $j<k$ or both $j=k$ and $w_{1} \leqq w_{2} \quad\left(w_{1}, w_{2} \in C_{m} ; j, k \in \mathbb{Z}\right)$. Therefore $L_{m}$ is an $l$-metabelian $o$-group. Indeed if $\mathbb{Q}(m)=\left\{r / m^{s}: r, s \in \mathbb{Z}\right\}$, an $l$-subgroup of the o-group $\mathbb{Q}$, and $\psi \in \operatorname{Aut}(\mathbb{Q}(m),+, 0, \leqq)$ is multiplication by $m$, then we have shown that $L_{m}$ is $l$-isomorphic to $\mathbb{Q}(m) \bar{x}\langle\psi\rangle$. It follows that $L\left(m_{1}\right)$ and $L\left(m_{2}\right)$ are not $l$-isomorphic if $m_{1} \neq m_{2}$ and the theorem is proved.

I know of no other examples of finitely presented l-groups that are o-groups. Therefore, the following questions remain:

(I) Is every finitely presented $l$-group that is an $o$-group in fact $l$-soluble?

(II) Is every finitely presented $l$-group that is an $l$-soluble $o$-group actually l-metabelian?

\section{REFERENCES}

1. A. Bigard, K. Keimel and S. Solfenstein, Groupes et Anneaux Réticulés (Lecture Notes in Math, No. 608, Springer-Verlag, Heidelberg, 1977).

2. P. M. Conn, Universal Algebra (Harper and Row, New York, 1965).

3. M. R. Darnel, A. M. W. Glass and A. H. Rhemtulla, Groups in which every right order is two sided, Archiv der Math. 53 (1989), 538-542.

4. A. M. W. Glass, Ordered Permutation Groups (London Math. Soc. Lecture Notes Series No. 55, Cambridge University Press, 1981).

5. A. M. W. GLASS, Generating varieties of lattice-ordered groups: approximating wreath products, Illinois J. Math. 30 (1986), 214-221.

Department of Mathematics and Statistics

Bowling Green State University

Bowling Green, OH 43403-0221

U.S.A. 